\title{
Effect of high nitrate vegetable juice supplementation on plasma nitrate and blood pressure in healthy adults: a pilot randomized crossover intervention in healthy volunteers
}

Nour Elsahoryi ( $\square$ nelsahoryi01@qub.ac.uk)

Queen's University Belfast

Christopher Cardwell

Queen's University Belfast

Sarah Gilchrist

Queen's University Belfast

Jayne Woodside

Queen's University Belfast

\section{Research Article}

Keywords: Beetroot, vegetable juice, nitrate, hypertension

Posted Date: March 25th, 2021

DOl: https://doi.org/10.21203/rs.3.rs-258007/v1

License: (c) (1) This work is licensed under a Creative Commons Attribution 4.0 International License.

Read Full License 


\section{Abstract}

Background: Beetroot juice has been demonstrated to decrease blood pressure due to the high inorganic nitrate content, but few studies have tested the effect of other high nitrate vegetable juices on blood pressure.

Methods: This pilot randomized crossover trial aimed to investigate the effect of two different high nitrate vegetable juices on plasma nitrate concentrations and blood pressure in healthy adults. Eighteen healthy volunteers were randomized to receive $115 \mathrm{ml}$ of beetroot juice or $250 \mathrm{ml}$ of green leafy vegetable juice for seven days. Blood samples were collected, and clinic blood pressure measured at baseline and at the end of each juice consumption. Daily home blood pressure assessment was conducted two hours after juice consumption. Nitrate and nitrite concentrations were analysed using a commercially available kit on a Triturus automated ELISA analyser. Hills and Armitage analysis was used for the two- period crossover design and paired sample t- tests were performed to compare within-group changes.

Results: Plasma nitrate and nitrite concentration significantly increased and there was significant reduction in clinic and home SBP mean during the beetroot juice period (P-values 0.004 and 0.002, respectively). Home DBP reduced significantly during green leafy vegetable juice consumption week (Pvalue 0.03 ). The difference between groups did not reach statistical significance during the formal crossover analysis adjusted for period effects.

Conclusion: Beetroot juice and green leafy vegetable juice may reduce systolic or diastolic blood pressure but there was no statistically significant difference between the two juices, although this was only a pilot study.

\section{Background}

Vegetables represent the main dietary source of nitrate and contribute $85 \%$ of daily nitrate intake, while other sources of nitrate come from other foods, such as meat and from water $(1,2)$. The amount of nitrate varies between vegetables and also depends on many factors, such as soil type, genotype, growth condition, storage and transport conditions $(1,2)$. For example: celery, cress, chervil, lettuce, red beetroot, spinach and rocket contain more than $250 \mathrm{mg}$ per $100 \mathrm{~g}$ of fresh weight, while broccoli, carrot, cauliflower, cucumber, pumpkin and chicory contain less than $20 \mathrm{mg}$ per $100 \mathrm{~g}$ of fresh weight (1). Many studies have suggested that inorganic nitrate can have health benefits on cardiovascular disease (CVD) in general and hypertension in particular via the nitrite- nitric oxide (NO) pathway (3-6). As beetroot is a rich source of dietary inorganic nitrate (7), a large number of intervention studies have demonstrated a positive effect of beetroot juice (BRJ) on BP and, therefore, CVD risk (8-13).

Most studies have focused on the acute effect of BRJ on BP $(9,13-19)$, while few studies have examined the effect of chronic nitrate consumption on BP (20-22). The first study that tested the chronic effects of sodium nitrate on BP was a crossover study in healthy volunteers with 3 days supplementation and 10 days washout (22). The results reported a significant reduction in diastolic blood pressure (DBP) by -3.7 
$\mathrm{mmHg}(22)$. Since then the interest in the effects of dietary nitrate on cardiovascular health has grown, and several studies have confirmed the lowering effects of dietary nitrate on chronic and acute BP, which have been reviewed (2). A systematic review and meta-analysis of 16 trials (both acute and chronic in design) suggested that high inorganic nitrate supplementation (inorganic nitrate supplementation and via increased beetroot or BRJ intake) reduced systolic blood pressure (SBP) by $-4.4 \mathrm{mmHg}$, while DBP was not affected, and the findings in this meta-analysis were homogeneous (7). A more recent systematic review and meta-analysis of nine crossover trials and three parallel trials also reported that inorganic nitrate and beetroot consumption produced beneficial effects on endothelial function (23). Another systematic review and meta-analysis of 34 studies, including seventeen of them measured acute BP and response to a single dose of inorganic nitrate, reported that inorganic nitrate intake significantly reduced SBP by $-4.8 \mathrm{mmHg}$ and DBP by $-1.74 \mathrm{mmHg}$; in addition such intake also improved endothelial function, reduced atrial stiffness and reduced platelet aggregation (24). Recently, Ocampo et al (2018) reported that BRJ intervention could significantly decrease the risk of suffering cardiovascular events and that it should be promoted as a key component of a healthy lifestyle to control blood pressure in healthy and hypertensive individuals (17). In contrast, the most recent systematic review and meta-analysis of 12 randomized control trials examining the effect of BRJ on BP concluded that there was no change either on SBP or DBP in the intervention groups compared with the control group (19), therefore there is still some inconsistency in the literature.

Although there are several other nitrate- rich food sources, including green leafy and root vegetables (25), research on the pharmacokinetic and physiological effects of nitrate supplementation has mainly used either sodium nitrate or BRJ as a nitrate donor. Very few studies have investigated the effect of more than one high nitrate sources $(10,26)$. A recent crossover study found that (beetroot, rocket salad or spinach) juice increased plasma nitrate and lowered BP to a greater extent than sodium nitrate (26), after a single exposure. A further crossover trial investigated the effect of a high nitrate diet and control diet on BP for 1 week (3). The high nitrate diet consisted of consumption of five different types of green leafy vegetables (salad, such as lettuce, rocket, celery, leeks, fennel and mixed salad leaves), while on the control arm, participants had to avoid these for seven days. The results for BP indicated that consuming two portions of a variety of high nitrate vegetables daily over $7 \mathrm{~d}$ reduced SBP (by $4 \mathrm{mmHg}$ ) in healthy, normotensive young women, but had no significant effect on DBP (3).

This study aimed to examine the feasibility of carrying out a randomised crossover intervention study of two different high nitrate vegetable juices, consumed over one week, on plasma nitrate levels and BP in healthy adults. In this study, we hypothesized that oral supplementation of BRJ and green leafy vegetable juice (GLVJ) with similar amounts of nitrate would decrease BP in healthy participants and that the effect on BP would be independent of the BP measurement protocol (clinic measurement and home measurement).

\section{Materials And Methods \\ Participants}


A total of 18 non-smoking subjects, between 30 and 65 years of age and with body mass index $(\mathrm{BMI})>$ $35 \mathrm{~kg} / \mathrm{m}^{2}$, were recruited between November 2016 and March 2017. Participants were excluded if they had medical conditions (i.e., diabetes, acute coronary syndrome or transient ischaemic attack within the past 3 months, using oral anticoagulation therapy), if they reported excessive alcohol consumption ( $>14$ $\mathrm{U} /$ week), if they were pregnant or lactating or had dietary restrictions that could interfere with the nutritional intervention and study outcomes. Written informed consent was obtained from all participants prior to participation in the study. The study was approved by the School of Medicine, Dentistry and Biomedical Sciences Research Ethics Committee of Queen's University Belfast and adhered to the guidelines contained within the Declaration of Helsinki.

\section{Study design}

The study was a one-week, randomized crossover trial (Figure 1). Participants were randomized either to BRJ or GLVJ by use of a block design (block size $n=4$; www.randomization.com) for 1 week, with a 2weeks washout between the two interventions.

The registration number is NCT04736784. The first trial registration date on the website is $03 / 02 / 2021$. Actual study start date: $30 / 1 / 2017$, Actual study completion date: $15 / 5 / 2017$. The source of the registration is ClinicalTrials.gov.

\section{Study Interventions}

The intervention was designed to give the participants equal amount of nitrate (approximately $340 \mathrm{mg}$ nitrate daily) from both juices. Therefore, participants were randomized to receive $115 \mathrm{ml}$ of BRJ or 250 $\mathrm{ml}$ of GLVJ daily for 7 days. Both juices were made in the UK. The amount of nitrate in the BRJ and in the leafy juice was calculated based on previous research $(1,27,28)$. The leafy vegetable juice contained $30 \%$ celery, 30\% cucumber, 15 romaine, $15 \%$ spinach, $5 \%$ kale, $2.5 \%$ lemon and 2.5 ginger. The calculated amount of nitrate in the GLVJ (340.30 mg per $250 \mathrm{ml}$ ) was less than the amount of the nitrate in BRJ $(735.75 \mathrm{mg} / 250 \mathrm{ml})$. Therefore, the participants were asked to drink $250 \mathrm{ml}$ of the GLVJ daily for 7 days and $115 \mathrm{ml} \mathrm{BRJ}$ daily for 7 days. Previous studies have suggested that the peak action time for dietary nitrate to reduce BP is $\sim 2.5-3 \mathrm{~h}$ after ingestion (29). Therefore, participants were requested to drink both juices in the evening, $2-3 \mathrm{~h}$ before measuring home BP.

Participants were advised to follow their usual diet and not to make any changes to dietary or lifestyle habits and instructed that their body weight was to remain stable for the duration of the intervention. They were also advised to avoid mouth wash during the study, as antibacterial mouthwash has been shown to attenuate the rise in plasma nitrite (3).

\section{Outcomes}

Height was assessed at baseline using a Leicester portable height measure. Before and after each intervention period, participants were weighed using recently calibrated digital weighing scales (Seca 876, 
SECA, Birmingham, UK), and BP was measured.

Questionnaires to assess physical activity and education level were completed at baseline. General physical activity was assessed (i.e. over the last 4 weeks) using the general practice physical activity questionnaire (GPPAQ). The primary end-point of this study was to compare the effect of two different high nitrate juices on clinic and home- measured BP between intervention groups (over the course of the 4- week intervention period).

\section{Blood pressure measurements}

\section{Clinic blood pressure measurements}

With the patient seated comfortably for 15 minutes prior to measurement and the arm supported at the level of the heart, clinical BP was measured in triplicate by using an automated BP monitor (Omron Healthcare, The Netherlands). The final value was calculated by the average from the 2 nd and 3 rd readings.

\section{Daily home blood pressure monitoring}

An automated BP monitor (Omron Healthcare, The Netherlands) was provided to each participant to measure daily resting BP at home. Participants were asked to conduct duplicate measurements, in a seated position, at evening, two hours after juice consumption and before going to bed. Participants were trained on how to use the monitor, with an emphasis on the correct positioning of the monitor and arm. In addition, written instructions on measuring BP were provided. A form was provided to participants to record the BP readings (SBP and DBP) and time of each measurement. The average of the measurements was calculated; the average of the values for each week was calculated to evaluate differences in BP between the two interventions. To increase participant compliance, text messages were sent to the participants, as a reminder, daily, during the course of the study before juice drinking to drink the juice and after juice drinking to record BP values, and other messages as a reminder before each lab visit. A total of $32 \mathrm{ml}$ non- fasting whole blood, collected into serum and EDTA tubes, was obtained from participants, using standard phlebotomy procedures before and after each intervention period. All participants were seated for venepuncture. Samples were centrifuged at $3000 \mathrm{rpm}$ for 15 minutes at $4^{\circ} \mathrm{C}$ and separated appropriately for the proposed assays. Sample aliquots were stored at $-80^{\circ} \mathrm{C}$ until analysis.

\section{Analysis of plasma nitrate and nitrite concentrations}

Plasma nitrate concentrations were quantified using a commercial kit and calibrators from R\&D Systems (Minneapolis, MN, USA) according to the manufacturers' instructions. Kits were run on a Triturus automated ELISA analyser supplied by Grifols UK Ltd. Plasma samples were filtered using Amicon Ultra 2, 10,000 MW centrifugal filter units (Millipore UK Ltd). The assay procedure measured total nitrite by converting nitrate to nitrite. Samples and standards, as well as quality controls, were prepared as per the 
manufacturer instructions. The plate was then incubated at room temperature for 10 minutes and samples were read at $540 \mathrm{~nm}$ with wavelength correction at $690 \mathrm{~nm}$.

\section{Participants' view of the intervention juices}

A series of 5- point Likert- scales were used to assess ease of consumption of the intervention juices in the following question: Based on your experience in this study, did you find the juice (either BRJ or GLVJ based on the intervention week): very easy, easy, sometimes hard sometimes easy, hard, very hard. The same question was asked to assess the acceptability, with the following response choices: very acceptable, acceptable, unsure, unacceptable, very unacceptable. In addition, participants were asked if drinking the juice for seven days is shown to help improve blood pressure: (a) I would be willing to continue drinking the juice after the study finished and (b) I would be willing to spend approximately $£ 2.25$ pound per week for BRJ or $£ 14.00$ per week for GLVJ and how much they agreed with the statements given, with the following responses: strongly agree, agree, unsure, disagree, strongly disagree.

In addition, open and closed questions were also used such as whether the participants found the study products gave any side effects and if they would be willing to continue taking the products postintervention, if found to be beneficial to health. For example, "Did you experience any side effects when drinking (juice name), during the study", "If you found drinking (juice name) over a period of seven days hard or unacceptable, please tell us why".

\section{Statistical analysis}

The data were checked for normality by using the Kolmogorov-Smirnov test. Descriptive statistics were obtained for all variables. Normal variables were reported as mean (SD) and categorical variables were reported as percentages. Descriptive statistics and paired t- test were performed using SPSS v17.0 for Windows (SPSS Inc, Chicago, IL). Paired sample t- tests were performed on BP data to compare the within-group effect of each juice before and after each intervention. Hills and Armitage analysis(30) used for two- period crossover design using STATA software (version 9.0, StataCorp, TX, USA) and Excel (Windows 2013). Treatment effects (difference in mean adjusted for period) and $95 \% \mathrm{Cl}$ were calculated. $P$ values $<0.05$ (2-tailed) were considered statistically significant.

\section{Results}

\section{Recruitment and baseline}

A total of 18 (12 females and 6 males) completed the study. Table 1 show the baseline characteristics for all study participants and separately according to assigned intervention group. Based on the randomization, eight participants started with BRJ and ten participants started with GLVJ. The mean age of the study participants was $40.9 \mathrm{y}$. The baseline physical activity category for most participants was moderate physical activity level. The education level was postgraduate/ high degree level for $61 \%$ of the participants. 


\section{Effect of BRJ versus GLVJ intervention on SBP, DBP and serum nitrate and nitrite concentrations}

As shown in Table 2, there was no statistically significant difference in change in clinic or home BP between BRJ and GLVJ, during the formal crossover analysis adjusted for period effects. The reduction in SBP and DBP was numerically higher during BRJ period but the difference between groups did not reach statistical significance. The results of Hills and Armitage analysis also indicated that there was an increase in nitrate and nitrite concentration during both interventions, but, again, there were no statistically significant differences between interventions. The mean difference in BMI, BP (Clinic and Home) and Nitrate during each intervention period is shown in table 3 . The results indicated that was no significant change in BMI during each intervention. For BP, the result indicated that there were statistically significant reductions in clinic and home SBP mean during the BRJ period (P-values 0.004 and 0.002, respectively), while the reduction in home DBP was statistically significant during the GLVJ intervention (P-value 0.03). Nitrate and nitrite concentrations increased significantly during both BRJ and GLVJ periods.

\section{Acceptability}

At the end of each intervention week, acceptability and willingness was assessed. The results indicated that most participants reported urine colour changes after drinking BRJ and bowel habit changes after drinking GLVJ, as shown in Table 4. However, none of the side effects mentioned were serious and none of the participants needed to seek medical advice or discontinue participation in the study. The majority of participants rated both juices as easy to consume. Participants who reported that GLVJ was unacceptable (5.6\%), and $16.7 \%$ reported that GLVJ was very unacceptable due to the taste and odd smell and due to changes in bowel habit. In both intervention 33.3\% (same percentage in both interventions) of the participants were willing to consume/purchase the study products post-intervention if the products could be demonstrated to improve heart health.

\section{Discussion}

This is the first study that has determined the effect of two different high nitrate juices on chronic BP, measured both as home BP and clinic BP. Our results indicated that, after BRJ consumption, home and clinic SBP reduced significantly while after GLVJ consumption, home DBP reduced significantly. The crossover analysis, however, indicated that there was no statistically significant difference between the BRJ and GLVJ interventions, although, as this was a pilot study, we were probably not powered to detect this.

These results agree with some crossover studies $(3,10)$ which compared home and clinic BP, our results are similar to one randomized trial results which compared three different methods to measure BP (Clinic BP, Daily home BP monitoring and 24-Hour ABPM) (10). The authors looked for the effect of BRJ and blackcurrant juice on BP for 24 non- smoking subjects. The results reported that BP measured by all methods reduced during the intervention, but that the changes in clinic BP, 24- Hour ABPM and Daily home BP monitoring were not statistically significant between the two interventions (10). Moreover, the 
results of this study agree with very recent intervention studies $(15,16)$. Researchers examined NO3supplementation on microvascular and large-vessel EF and BP in a randomized, double-blind, placebocontrolled pilot study in healthy older adults and they concluded that BRJ ingestion potentially improves $\mathrm{BP}$ and large-vessel EF in healthy older adults (15). Acute BR supplementation increased plasma nitrite concentrations and reduced SBP and DBP in both older and younger adults in a double-blind, crossover study (16).

Using clinic BP is the most commonly used method, but its poor reliability is universally recognized, due to measurement bias associated with white- coat syndrome, standardization of protocol, and operator bias (31). Home BP readings may provide more reproducible results due to a lack of white- coat syndrome (32) but, on the other hand, home BP monitors do not provide nocturnal recordings and therefore cannot give information on diurnal patterns in BP, which are more prevalent to the cardiovascular risk factors and the accuracy of devices remains a limiting factor associated with home BP monitors (33). Therefore, measuring BP in continuous basis (ambulatory BP monitoring) can detect abnormal fluctuations in BP that might go unnoticed when BP is only measured in the doctor's office. Our trial examined the effect of two high nitrate juices on BP in healthy individuals in the absence of dietary restrictions, such as a low nitrate diet. Dietary restrictions in studies with high nitrate juice and BP remove confounding dietary factors that may have an effect (negative or positive) on BP, thus making interpretation of study results more straightforward. The drawback, however, is that BRJ and GLVJ as a nutritional intervention to regulate BP would likely be consumed as part of a normal diet, not as part of a low nitrate diet or in the absence of other dietary components (i.e. coffee, alcohol, black tea) that may affect BP (13). It would therefore be uncertain whether there is any clinically relevant benefit from BRJ or GLVJ on BP in the unregulated home environment.

Comparing between the results after and before each intervention, the results of BRJ agreed with many previous studies that support the hypothesis that a high nitrate diet, mainly as BRJ, can reduce BP. A recent systematic review and meta-analysis (2018) of 11 randomized control trials reported that BRJ has a positive effect in reducing BP compared with control group, mainly on SBP (17)... An older metaanalysis of 16 trials showed that inorganic nitrate and BRJ supplementation was associated with a significant reduction in SBP, whereas no significant effect was observed for diastolic BP (7). In addition, a review of human intervention studies involved studies in the chronic and acute setting and reported that there was an inverse relationship between dose of nitrate consumed and corresponding reduction in SBP (by $3 \mathrm{mmHg}$ with doses of nitrate as low as $3 \mathrm{mmol}$ of nitrate) in addition to the beneficial effects of dietary nitrate on endothelial function (23). However, in contrast, Remington and Winters (2019) reported that there was no effect of BRJ on BP in 12 randomized control trials (19). For GLVJ, to our knowledge, this is the first study determine the effect of such a juice on BP in a chronic setting. The juices given should have had similar nitrate content, but may have differed in levels of other nutrients, such as betaine, which could impact on BP-lowering potential. The findings show some effects of GLVJ on BP (home DBP), but a potential lack of power to detect differences between the BRJ and GLVJ juice groups means it is difficult to draw conclusions from this pilot study. Therefore, more studies are required in this area, and data from this study can be used to power these appropriately. Finally, in response to the levels 
of liking questionnaire, participants generally rated their liking of both juices acceptable and easy to consume. Both interventions were well tolerated. In addition, most participants were willing to consume them and purchase them if studies demonstrate the beneficial effects of either juice on cardiovascular health including BP. The most commonly self- reported adverse effect with BRJ was red urine (beeturia) and this agreed with other beetroot studies $(10,34)$. Some volunteers experienced mild, temporary abdominal discomfort from GLVJ, however, no serious adverse events were reported.

\section{Strengths And Limitations}

There were several strengths in this pilot study. Firstly, general strengths were: it was a crossover randomised intervention; BRJ and GLVJ were supplied to participants to ensure limited costs were incurred by the participants; and advice and support was given at the beginning and throughout the study to ensure participants understood fully the requirements for involvement. Secondly, text messages were used to encourage good participant compliance and to monitor compliance and minimise dropouts over the course of the study: for example, daily messages were sent before juice drinking to drink the juice and after juice drinking to record BP values, and other messages were sent as a reminder before each lab visit. Finally, participants had no restrictions on their usual diet; therefore, requirements for the study were not intensive so it would have been easy for participants to stick to their usual routine and not have too many demands imposed by the study protocol. On the other hand, this study also had limitations: first, the small sample size and short duration of this trial may have reduced the power of the study to detect significant differences in change in BP between the intervention groups, although it was designed as a pilot study. Secondly, participants may have forgotten to drink the juice or drunk less than the appropriate amount, despite efforts to maximise compliance. Third, when deciding on how much juice each participant should drink, we used nitrate content from the literature which shows marked variation and did not measure the nitrate content of the juices directly. Therefore, nitrate concentration estimates were potentially unreliable. Fourth, the juice volume was not the same, which may have affected the fluid volume in the body or the concentration of some nutrients. Finally, the randomization was not stratified for gender, and some trials of beetroot and BP reported a relationship between gender and the effect of beetroot on $\operatorname{BP}(9,13,20)$.In addition, although we did not observe any acute serious adverse effects, a few participants reported minor gastrointestinal complaints after ingestion of the juices. However, this was only a pilot study therefore it may be helpful to perform a retrospective power calculation to inform a larger study and conduct it for a longer time frame to allow for further investigation.

\section{Conclusions}

In conclusion, analysis of a two-period crossover trial testing consumption of BRJ and GLVJ by healthy volunteers for 7 days indicated that there was no significant difference in change in BP between the two juices in both periods. In addition, within group analysis of the results indicated that clinic and home SBP reduced significantly after BRJ consumption and home DBP reduced significantly after GLVJ consumption. Based on the acceptance and tolerability assessment, this study approved the feasibility of 
carrying a larger, appropriately powered intervention study to investigate the effect of the used juices (BRJ and GLVJ) on BP as tolerability was overall acceptable for both juices during the intervention. Additional studies with high nitrate vegetables juice in larger groups of healthy people are needed to determine whether they affect BP and explore mechanism of action.

\section{List Of Abbreviations}

BP: Blood pressure;

SBP: Systolic blood pressure;

DBP: Diastolic blood pressure;

BRJ: Beetroot juice;

GLVJ: Green leafy vegetable juice.

\section{Declarations}

\section{Ethical approval and consent to participate}

All participants in this study gave written informed consent and the study had the approval of the School of Medicine, Dentistry and Biomedical Sciences research ethics committee at Queen's University Belfast and adhered to the guidelines contained within the Declaration of Helsinki.

\section{Consent for publication}

Not applicable

\section{Availability of data and materials}

The datasets used and/or analysed during the current study are available from the corresponding author on reasonable request.

\section{Competing interests}

The authors declare that they have no competing interests.

\section{Funding}

The funding of this trial was provided by Queen's University Belfast, UK and Petra University, Jordan.

\section{Authors' contributions}


The first and last authors designed the study, analysed the data and had primary responsibility for final content. The first author recruited the participants, collected all data, statistically analysed the data and drafted the manuscript. The second author supported the statistical analysis. The third author supported the fieldwork and completed and the laboratory analysis. All authors approved the final manuscript.

\section{Acknowledgements}

The authors would like to acknowledge the Jordanian Petra University (the sponsor of the PhD studentship) and all the study volunteers who participated in this study.

\section{References}

1. Hord NG, Tang Y, Bryan NS. Food sources of nitrates and nitrites: the physiologic context for potential health benefits. The American journal of clinical nutrition. $2009 \mathrm{Jul} ; 90(1): 1-10$.

2. Hobbs DA, George TW, Lovegrove JA. The effects of dietary nitrate on blood pressure and endothelial function: a review of human intervention studies. Nutrition research reviews. 2013 Dec;26(2):210-22.

3. Ashworth A, Mitchell K, Blackwell JR, Vanhatalo A, Jones AM. High-nitrate vegetable diet increases plasma nitrate and nitrite concentrations and reduces blood pressure in healthy women. Public health nutrition. 2015;18(10):1-10.

4. Hung H-C, Joshipura KJ, Jiang R, Hu FB, Hunter D, Smith-Warner SA, et al. Fruit and vegetable intake and risk of major chronic disease. Journal of the National Cancer Institute. 2004 Nov 3;96(21):157784.

5. Joshipura KJ, Hu FB, Manson JE, Stampfer MJ, Rimm EB, Speizer FE, et al. The effect of fruit and vegetable intake on risk for coronary heart disease. Annals of internal medicine. 2001 Jun 19;134(12):1106-14.

6. Ness AR, Chee D, Elliott P. Vitamin $C$ and blood pressure-an overview. Journal of human hypertension. 1997 Jun;11(6):343-50.

7. Siervo M, Lara J, Ogbonmwan I, Mathers JC. Inorganic nitrate and beetroot juice supplementation reduces blood pressure in adults: a systematic review and meta-analysis. The Journal of nutrition [Internet]. 2013 Jun;143(6):818-26. Available from: http://www.ncbi.nlm.nih.gov/pubmed/23596162

8. Bondonno CP, Liu AH, Croft KD, Ward NC, Shinde S, Moodley Y, et al. Absence of an effect of high nitrate intake from beetroot juice on blood pressure in treated hypertensive individuals: A randomized controlled trial. American Journal of Clinical Nutrition. 2015;102(2):368-75.

9. Webb AJ, Patel N, Loukogeorgakis S, Okorie M, Aboud Z, Misra S, et al. Acute blood pressure lowering, vasoprotective, and antiplatelet properties of dietary nitrate via bioconversion to nitrite. Hypertension. 2008 Mar;51(3):784-90.

10. Jajj, Rennie K, Brandt K, Qadir O, Siervo M. Beetroot supplementation lowers daily systolic blood

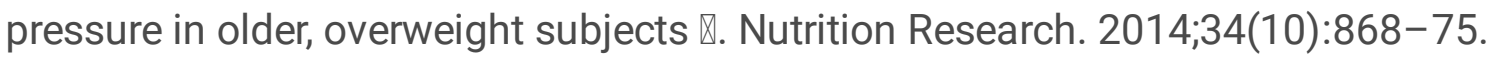


11. Sobko T, Marcus C, Govoni M, Kamiya S. Dietary nitrate in Japanese traditional foods lowers diastolic blood pressure in healthy volunteers. Nitric oxide: biology and chemistry / official journal of the Nitric Oxide Society. 2010 Feb 15;22(2):136-40.

12. Gee LC, Ahluwalia A. Dietary Nitrate Lowers Blood Pressure: Epidemiological, Pre-clinical Experimental and Clinical Trial Evidence. Current Hypertension Reports. 2016 Feb 27;18(2):17.

13. Coles LT, Clifton PM. Effect of beetroot juice on lowering blood pressure in free-living, disease-free adults: a randomized, placebo-controlled trial. Nutrition journal. 2012;11(1):106.

14. Raubenheimer K, Hickey D, Leveritt M, Fassett R, Ortiz de Zevallos Munoz J, Allen JD, et al. Acute Effects of Nitrate-Rich Beetroot Juice on Blood Pressure, Hemostasis and Vascular Inflammation Markers in Healthy Older Adults: A Randomized, Placebo-Controlled Crossover Study. Nutrients. 2017 Nov 22;9(11).

15. Jones, Dunn, Macdonald, Kubis, McMahon, Sandoo. The Effects of Beetroot Juice on Blood Pressure, Microvascular Function and Large-Vessel Endothelial Function: A Randomized, DoubleBlind, Placebo-Controlled Pilot Study in Healthy Older Adults. Nutrients [Internet]. 2019 Aug 2;11(8):1792. Available from: https://www.mdpi.com/2072-6643/11/8/1792

16. Stanaway L, Rutherfurd-Markwick K, Page R, Wong M, Jirangrat W, Teh KH, et al. Acute Supplementation with Nitrate-Rich Beetroot Juice Causes a Greater Increase in Plasma Nitrite and Reduction in Blood Pressure of Older Compared to Younger Adults. Nutrients [Internet]. $2019 \mathrm{Jul}$ 22;11(7):1683. Available from: https://www.mdpi.com/2072-6643/11/7/1683

17. Bonilla Ocampo D, Paipilla A, Marín E, Vargas-Molina S, Petro J, Pérez-Idárraga A. Dietary Nitrate from Beetroot Juice for Hypertension: A Systematic Review. Biomolecules [Internet]. 2018 Nov 2;8(4):134. Available from: http://www.mdpi.com/2218-273X/8/4/134

18. Shepherd Al, Costello JT, Bailey SJ, Bishop N, Wadley AJ, Young-Min S, et al. "Beet" the cold: beetroot juice supplementation improves peripheral blood flow, endothelial function, and anti-inflammatory status in individuals with Raynaud's phenomenon. Journal of applied physiology (Bethesda, Md: 1985) [Internet]. 2019;127(5):1478-90. Available from:

http://www.ncbi.nlm.nih.gov/pubmed/31343948

19. Remington J, Winters K. Effectiveness of dietary inorganic nitrate for lowering blood pressure in hypertensive adults. JBI Database of Systematic Reviews and Implementation Reports [Internet]. 2019 Mar;17(3):365-89. Available from: http://journals.Iww.com/01938924-201903000-00008

20. Vanhatalo A, Bailey SJ, Blackwell JR, DiMenna FJ, Pavey TG, Wilkerson DP, et al. Acute and chronic effects of dietary nitrate supplementation on blood pressure and the physiological responses to moderate-intensity and incremental exercise. American journal of physiology Regulatory, integrative and comparative physiology. 2010 Oct;299(4):R1121-31.

21. Bailey SJ, Winyard P, Vanhatalo A, Blackwell JR, Dimenna FJ, Wilkerson DP, et al. Dietary nitrate supplementation reduces the 02 cost of low-intensity exercise and enhances tolerance to highintensity exercise in humans. Journal of applied physiology (Bethesda, Md: 1985). 2009 Oct;107(4):1144-55. 
22. Larsen FJ, Ekblom B, Sahlin K, Lundberg JO, Weitzberg E. Effects of dietary nitrate on blood pressure in healthy volunteers. The New England journal of medicine. 1996 Dec 28;355(26):2792-3.

23. Lara J, Ashor AW, Oggioni C, Ahluwalia A, Mathers JC, Siervo M. Effects of inorganic nitrate and beetroot supplementation on endothelial function: a systematic review and meta-analysis. European journal of nutrition. 2016 Mar;55(2):451-9.

24. Jackson JK, Patterson AJ, MacDonald-Wicks LK, Oldmeadow C, McEvoy MA. The role of inorganic nitrate and nitrite in cardiovascular disease risk factors: a systematic review and meta-analysis of human evidence. Nutrition reviews. 2018 May 1;76(5):348-71.

25. Ysart G, Miller P, Barrett G, Farrington D, Lawrance P, Harrison N. Dietary exposures to nitrate in the UK. Food additives and contaminants. 1999 Dec;16(12):521-32.

26. Jonvik KL, Nyakayiru J, Pinckaers PJ, Senden JM, van Loon LJ, Verdijk LB. Nitrate-Rich Vegetables Increase Plasma Nitrate and Nitrite Concentrations and Lower Blood Pressure in Healthy Adults. The Journal of nutrition. 2016;146(5):986-93.

27. Alexander J, Benford D, Cockburn A, Cravedi J, Dogliotti E, Domenico A Di, et al. Nitrate in vegetables Scientific Opinion of the Panel on Contaminants in the Food chain. EFSA Journal. 2008 Jun;6(6):689.

28. Bahadoran Z, Mirmiran P, Jeddi S, Azizi F, Ghasemi A, Hadaegh F. Nitrate and nitrite content of vegetables, fruits, grains, legumes, dairy products, meats and processed meats. Journal of Food Composition and Analysis. 2016 Aug;51:93-105.

29. Lidder S, Webb AJ. Vascular effects of dietary nitrate (as found in green leafy vegetables and beetroot) via the nitrate-nitrite-nitric oxide pathway. British journal of clinical pharmacology. 2013 Mar;75(3):677-96.

30. Hills M, Armitage P. The two-period cross-over clinical trial. British journal of clinical pharmacology. $1979 \mathrm{Jul} ; 8(1): 7-20$.

31. Beevers G, Lip GY, O’Brien E. ABC of hypertension. Blood pressure measurement. Part Isphygmomanometry: factors common to all techniques. BMJ (Clinical research ed). 2001 Apr $21 ; 322(7292): 981-5$.

32. Parati G, Stergiou GS, Asmar R, Bilo G, de Leeuw P, Imai Y, et al. European Society of Hypertension practice guidelines for home blood pressure monitoring. Journal of human hypertension. 2010 Dec;24(12):779-85.

33. George J, MacDonald T. Home Blood Pressure Monitoring. European Cardiology Review [Internet]. 2015;10(2):95. Available from: https://www.ecrjournal.com/articles/home-blood-pressure-monitoring

34. Kapil V, Khambata RS, Robertson A, Caulfield MJ, Ahluwalia A. Dietary nitrate provides sustained blood pressure lowering in hypertensive patients: A randomized, phase 2, double-blind, placebocontrolled study. Hypertension. 2015;65(2):320-7.

\section{Tables}


able 1. Baseline characteristics of participants randomized to BRJ and GLVJ.

\section{irst juice}

ge

Iale (\%)

emale (\%)

MI baseline BRJ $\left(\mathrm{kg} / \mathrm{m}^{2}\right)$

MI baseline GLVJ $\left(\mathrm{kg} / \mathrm{m}^{2}\right)$

ab SBP baseline BRJ ( $\mathrm{mm} \mathrm{Hg}$ )

ab DBP baseline BRJ (mm Hg)

ab SBP baseline GLVJ ( $\mathrm{mm} \mathrm{Hg}$ )

ab DBP baseline GLVJ ( $\mathrm{mm} \mathrm{Hg}$ )

ome SBP baseline BRJ ( $\mathrm{mm} \mathrm{Hg}$ )

ome DBP baseline BRJ ( $\mathrm{mm} \mathrm{Hg}$ )

ome SBP baseline GLVJ ( $\mathrm{mm} \mathrm{Hg}$ )

ome DBP baseline GLVJ ( $\mathrm{mm} \mathrm{Hg}$ )

hysical level
BRJ First $(n=8) \quad$ GLVJ First $(n=10)$

$45.25 \pm 12.71$

1 (5.6\%)

17 (94.4)

$26.45 \pm 4.89$

$26.68 \pm 4.86$

$116.13 \pm 10.18$

$69.88 \pm 6.01$

$111.44 \pm 10.04$

$64.81 \pm 6.43$

$109.02 \pm 13.62$

$65.72 \pm 6.02$

$105.68 \pm 12.02$

$67.71 \pm 6.16$
$37.40 \pm 4.55$

$10(55.6 \%)$

8 (44.4\%)

$24.84 \pm 3.58$

$24.82 \pm 3.61$

$122.30 \pm 11.36$

$76.35 \pm 8.61$

$123.85 \pm 13.51$

$80.45 \pm 6.47$

$119.88 \pm 10.5$

$73.27 \pm 6.95$

$118.39 \pm 10.33$

$70.92 \pm 6.87$

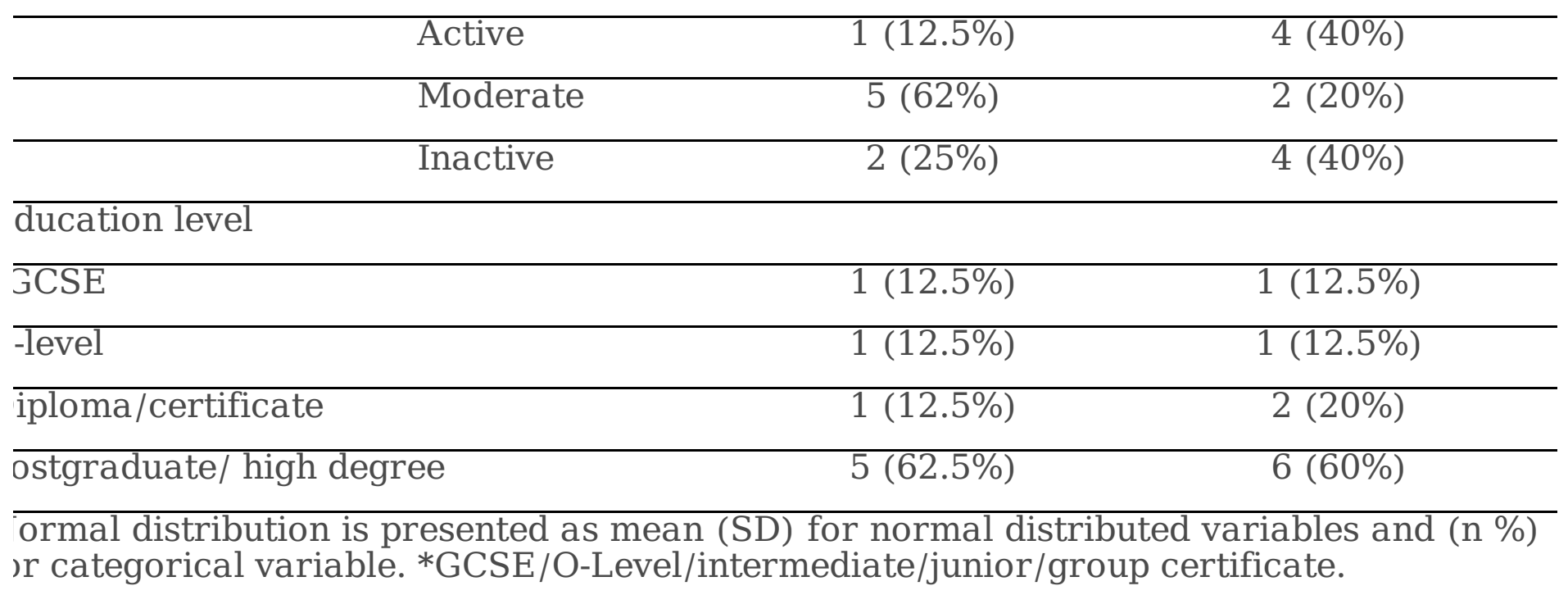


Table 2. The effect of BRJ and GLVJ on the clinic BP, nitrate for the healthy adults

\begin{tabular}{|c|c|c|c|c|c|}
\hline$\overline{\mathrm{BP}}$ & BRJ & GLVJ & Treatment effect* & $95 \% \mathrm{CI}$ & $\begin{array}{c}\text { P- } \\
\text { value }\end{array}$ \\
\hline & $\begin{array}{c}\text { Mean } \\
\text { change } \\
\text { (SD) }\end{array}$ & $\begin{array}{l}\text { Mean change } \\
\text { (SD) }\end{array}$ & $\begin{array}{c}\text { Mean difference in } \\
\text { change }\end{array}$ & & \\
\hline $\begin{array}{l}\text { Clinic SBP } \\
(\mathrm{mmHg})\end{array}$ & $\begin{array}{l}-6.06 \pm \\
7.76\end{array}$ & $-1.89 \pm 12.60$ & -4.83 & $\begin{array}{c}-11.56 \\
1.90\end{array}$ & 0.15 \\
\hline $\begin{array}{l}\text { Clinic DBP } \\
(\mathrm{mmHg})\end{array}$ & $\begin{array}{l}-2.14 \pm \\
8.42\end{array}$ & $0.92 \pm 7.83$ & -3.62 & $\begin{array}{l}-8.56 \\
1.33\end{array}$ & 0.14 \\
\hline $\begin{array}{l}\text { Home SBP } \\
(\mathrm{mmHg})\end{array}$ & $\begin{array}{l}-3.93 \pm \\
12.94\end{array}$ & $-8.41 \pm 14.57$ & -2.76 & $\begin{array}{l}-9.15 \\
3.65\end{array}$ & 0.38 \\
\hline $\begin{array}{l}\text { Home DBP } \\
(\mathrm{mmHg})\end{array}$ & $\begin{array}{l}-1.40 \pm \\
5.29\end{array}$ & $3.25 \pm 5.18$ & 3.27 & $\begin{array}{l}-0.25 \\
6.80\end{array}$ & 0.07 \\
\hline $\begin{array}{l}\text { Nitrate } \\
\text { (umol/L) }\end{array}$ & $\begin{array}{l}20.18 \pm \\
9.95\end{array}$ & $17.34 \pm 3.65$ & -7.24 & $\begin{array}{c}-19.91 \\
5.44\end{array}$ & 0.24 \\
\hline $\begin{array}{l}\text { Nitrite } \\
\text { (umol/L) }\end{array}$ & $\begin{array}{l}4.09 \pm \\
6.07\end{array}$ & $0.65 \pm 19.07$ & -6.75 & $\begin{array}{c}-19.49 \\
5.99\end{array}$ & 0.28 \\
\hline
\end{tabular}

*Treatment effect was adjusted for period. 
Table 3. The mean change in BMI, BP (clinic and home), nitrate and nitrite for participants randomized for BRJ and GLVJ

\begin{tabular}{|c|c|c|c|c|c|c|}
\hline & Pre BRJ & Post BRJ & & Pre GLVJ & Post GLVJ & \\
\hline & $\begin{array}{c}\text { Mean } \\
(\mathrm{SD})\end{array}$ & $\begin{array}{l}\text { Mean } \\
(\mathrm{SD})\end{array}$ & $\begin{array}{c}\mathrm{P}- \\
\text { value }\end{array}$ & $\begin{array}{c}\text { Mean } \\
\text { (SD) }\end{array}$ & $\begin{array}{c}\text { Mean } \\
(\mathrm{SD})\end{array}$ & $\begin{array}{c}\mathrm{P}- \\
\text { value }\end{array}$ \\
\hline $\begin{array}{l}\text { BMI } \quad(k g / \\
\left.m^{2}\right)\end{array}$ & $25.56 \pm 0.98$ & $25.62 \pm 0.96$ & 0.32 & $25.65 \pm 0.99$ & $25.64 \pm 0.97$ & 0.88 \\
\hline Clinic SBP & $\begin{array}{c}119.56 \pm \\
11.00\end{array}$ & $113.50 \pm 9.54$ & 0.004 & $\begin{array}{c}118.33 \pm \\
13.36\end{array}$ & $\begin{array}{c}118.69 \pm \\
11.64\end{array}$ & 0.92 \\
\hline Clinic DBP & $73.47 \pm 8.06$ & $71.33 \pm 6.26$ & 0.30 & $\begin{array}{c}73.50 \pm \\
10.15\end{array}$ & $74.42 \pm 6.61$ & 0.63 \\
\hline Home SBP & $\begin{array}{c}115.05 \pm \\
12.87\end{array}$ & $110.47 \pm 13.08$ & 0.002 & $\begin{array}{c}112.74 \pm \\
12.58\end{array}$ & $\begin{array}{c}112.43 \pm \\
11.23\end{array}$ & 0.90 \\
\hline Home DBP & $69.91 \pm 7.45$ & $69.08 \pm 8.12$ & 0.49 & $69.49 \pm 6.58$ & $66.89 \pm 7.13$ & 0.03 \\
\hline Nitrate & $13.78 \pm 4.80$ & $35.68 \pm 23.18$ & $\stackrel{\leq}{\leq}$ & $12.11 \pm 3.25$ & $\begin{array}{c}30.46 \pm \\
12.25\end{array}$ & $\begin{array}{l}\leq \\
0.001\end{array}$ \\
\hline Nitrite & $14.06 \pm 4.79$ & $35.83 \pm 23.14$ & $\begin{array}{c}\leq \leq \\
0.001\end{array}$ & $12.41 \pm 3.29$ & $\begin{array}{c}30.74 \pm \\
12.25\end{array}$ & $\begin{array}{l}\leq \\
0.001\end{array}$ \\
\hline
\end{tabular}

Statistical significance was determined by paired sample t- test. BP unit is mmHg. Nitrate and nitrite unit is umol/L. 
Table 4: Responses to tolerability of study products and willingness to consume/purchase post- intervention, according to juice type

\section{BRJ GLVJ \\ (\%) \\ (\%)}

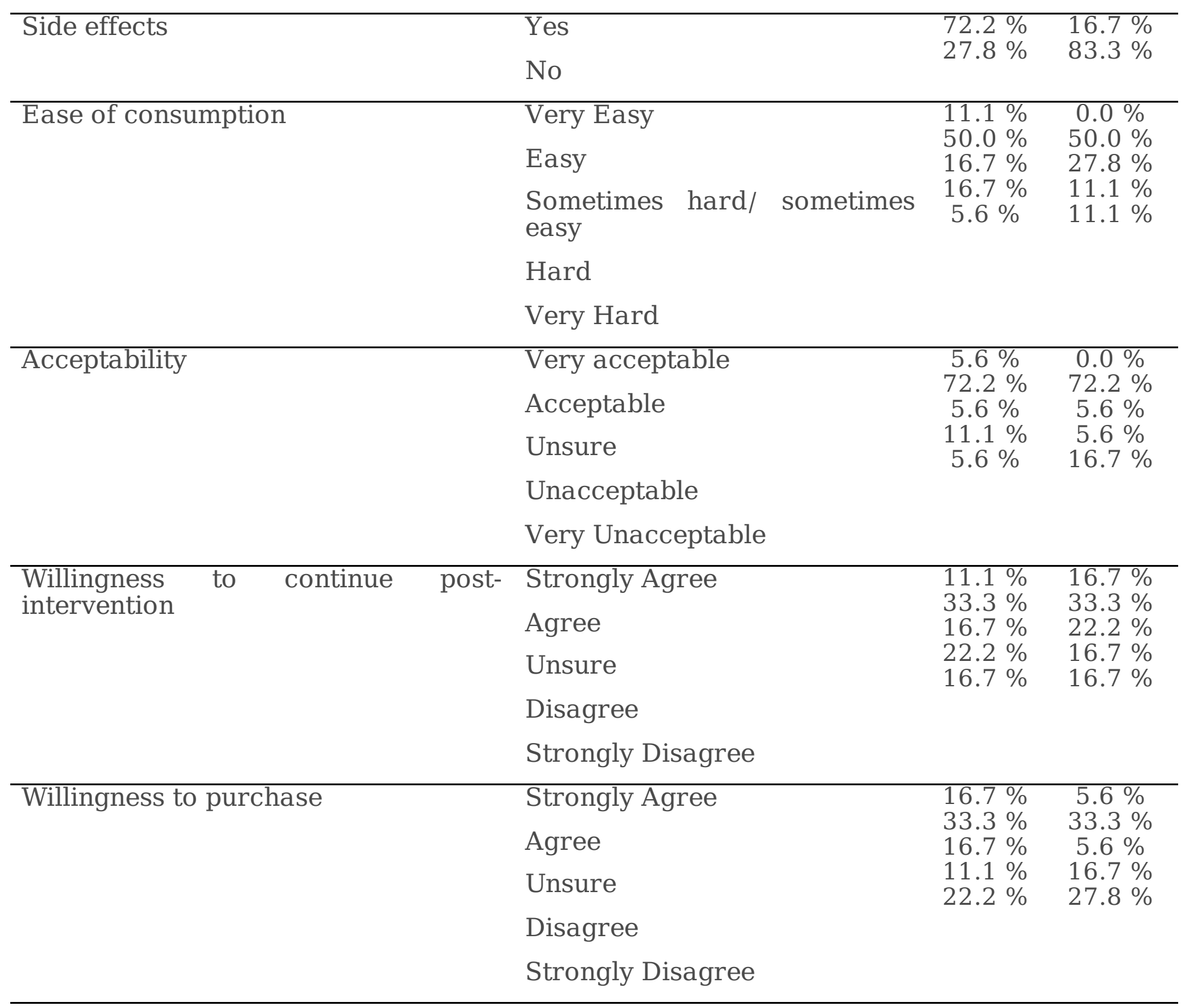

\section{Figures}




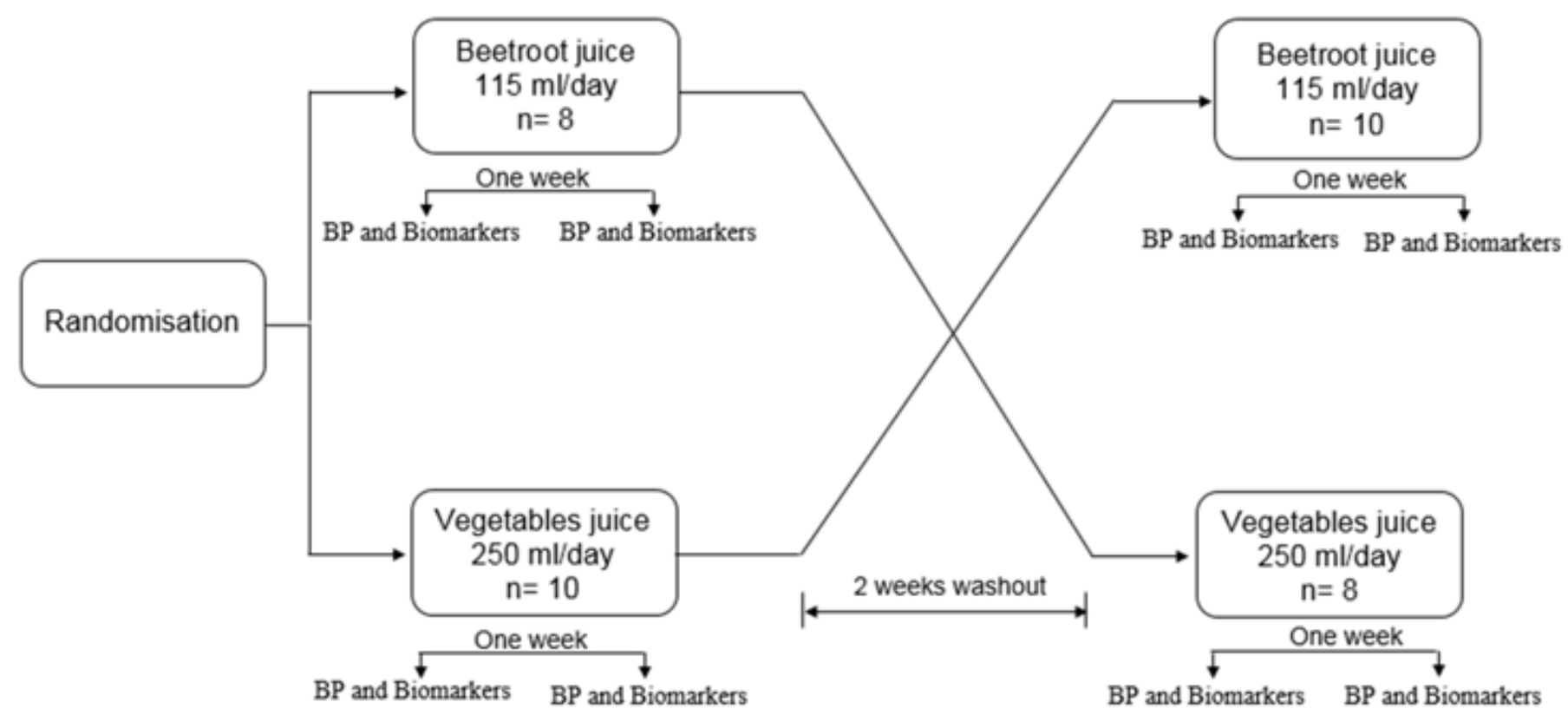

Figure 1

Overview of study design

\section{Supplementary Files}

This is a list of supplementary files associated with this preprint. Click to download.

- CONSORT2010ChecklistforNIVJUS.doc

- studyprotocol.docx 